\title{
INJERTO MICROVASCULARIZADO DE PERONÉ E IMPLANTES DENTALES. PARA RECONSTRUCCIÓN MANDIBULAR. PRESENTACIÓN DE UN CASO.
}

REINOSO-QUEZADA SANTIAGO*

Recibido: 08/11/2015

WINTERGERST-FISCH ALBERTO**

Aceptado: 18/11/2015

\section{RESUMEN}

La reconstrucción mandibular con injertos microvascularizados y rehabilitación protésica ha sido ampliamente mencionada en la literatura, sin embargo la utilización de los mismos de manera secundaria a la resección y más aún, de manera tardía, presenta retos importantes para el manejo y tratamiento de los pacientes que han sido mutilados; por lo que la reconstrucción involucra múltiples procedimientos quirúrgicos y sitios donadores más amplios lo que aumenta de manera significativa la morbilidad.

Palabras Clave. Injerto microvascular, Implantes dentales, reconstrucción mandibular

\section{ABSTRACT}

The microvascular grafts used for mandibular reconstruction and future prosthetic rehabilitation has been widely mentioned in the literature, however the use of these grafts in a secondary and late reconstruction provides important challenges for management and treatment for mutilated patients, so the maxilo-mandibular reconstruction involves several surgical procedures and large donor sites that consecuently rise the mordity rate.

Key words. Microvascular Grafts, dental implants, mandibular reconstruction

\section{PRESENTACIÓN DEL CASO}

Se presenta un caso de un paciente femenino de 49 años de edad que acude a consulta para ser valorada posterior a hemimandibulectomía izquierda realizada hace un año sin recibir tratamiento reconstructivo alguno. La paciente presenta un importante colapso de tejidos blandos faciales a nivel de hemicara izquierda. Fig 1 y 2. La apertura oral se encuentra conservada y el cóndilo contralateral se aprecia funcional y sin alteraciones estructurales. Intraoralmente el piso de boca y tegumentos se encuentran ligeramente desplazados hacia el lado contralateral Fig. 3. así como se observa cicatriz en cuello por abordaje quirúrgico cervical utilizado para la resección.

Se realiza tomografía computarizada en la cual se observa segmento mandibular izquierdo remanente ligeramente desplazado lateralmente por la tracción muscular ipsilateral. Fig 4 y 5 . La paciente presenta depresión por la profunda carga emocional que le ha provocado la secuela del manejo quirúrgico realizado. Se decide la realización de un procedimiento consistente en:

1. Primer tiempo quirúrgico toma y colocación de injerto osteomiocutáneo libre microvascularizado de peroné en región mandibular con colocación de placa de reconstrucción de Marx y prótesis articular. Fig. 6 y 7.

2. Segundo tiempo quirúrgico a los 6 meses del primero, colocación de implantes dentales osteointegrados. Fig. 10 y 11.

3. Colocación de prótesis implantomucosoportada 6 meses posterior a la colocación de implantes.

El procedimiento de toma y anastomosis del injerto microvascularizado de peroné estuvo a cargo de Ignacio Lugo, Cirujano Plástico, Fig. 6, es en este momento que se decide no realizar la colocación de la prótesis condilar ya que el tejido se encuentra sumamente colapsado en la región preauricular y además la probable disección del tejido podría alterar o lesionar el nervio facial. Culminado el mismo se mantiene en control postoperatorio a la paciente durante 6 meses hasta que se encuentre en condiciones ideales para la colocación de implantes dentales. Fig, 8 y 9.

Luego de seis meses se programa el procedimiento quirúrgico consistente en colocación de implantes dentales a nivel del segmento mandibular y del injerto del peroné micro vascularizado. Fig. 10 y 11 . el mismo que se culmina sin incidentes. Mantenemos en control postoperatorio durante 6 meses al paciente, actualmente el paciente se encuentra en la fase protésica y la construcción de la prótesis implato soportada a cargo del servicio de prótesis máxilofacial.

\footnotetext{
* Docente Especialista Cirugía Maxilofacial, Universidad Católica de Cuenca.Ecuador
}

** Cirujano Maxilofacial, Universidad Nacional Autónoma de México.México 


\section{PRINCIPIOS DE RECONSTRUCCIÓN ÓSEA.}

Existen muchas formas disponibles para reconstruir una deficiencia o un defecto óseo de la anatomía del esqueleto facial.

Estas incluyen injertos óseos, proteína morfogenética recombinante 2 y distracción osteogénica. Tabla 1.

Tabla.1. Clasificación de injertos óseos.

\begin{tabular}{lll}
\hline$\bullet$ & \multicolumn{2}{l}{ Autógeno } \\
& $\circ$ & Libres \\
& - & Corticales \\
& - & Medulares \\
& & Corticomedulares \\
& 0 & Microvascularizados \\
\hline$\bullet$ & Alogénico (Homologo) \\
& 0 & Seco congelado \\
& 0 & Descalcificado \\
\hline
\end{tabular}

Estas técnicas pueden ser usadas solas o en combinación; pudiendo ser beneficiadas con la aplicación de técnicas de fijación interna rígida y regeneración ósea guiada en caso de requerirse. ${ }^{1}$

La elección de una técnica de reconstrucción es influenciada por muchas variables que incluyen la localización, relación y dimensión del defecto, es muy importante evaluar las dimensiones del hueso subyacente así como disponibilidad y viabilidad de tejido blando y se deben conocer cuáles son los objetivos funcionales y estéticos que se quiere alcanzar.

Más allá de elegir una técnica de reconstrucción, se deben considerar las propiedades intrínsecas de los huesos faciales y su crecimiento natural y las características de remodelación.

Para que un hueso crezca o se regenere en áreas de presión directa, este debe de tener un proceso de reemplazo endocondral como el que ocurre en los huesos largos o en el cóndilo. ${ }^{2}$

Las áreas del esqueleto que son sometidas a presión, deben ser cubiertas por cartílago el cual es un tejido adaptado a esta función, crece intersticialmente, es mínimamente calcificado, avascular, nutrido por difusión. En contraste el hueso no puede soportar presión significativa porque la presión cerraría la cama vascular del periostio y la matriz ósea que es calcificada, debe estar vascularizada para poder crecer regenerarse 0 mantenerse, además la calcificación de la matriz permite el crecimiento intersticial, así que el hueso solo puede crecer mediante actividad aposicional de sus membranas.

El periostio tiene un componente de tejido conectivo denso y está estructuralmente adaptado para transferir fuerzas tensiles que son generadas por músculos, tendones y ligamentos al hueso.

La mayoría del esqueleto facial no está sujeto a carga durante su desarrollo, por esto no requiere de una fase endocondral así que se desarrolla por un proceso intramembranoso. En el estado natural el hueso es protegido de las cargas por la dentición y activamente recibe estimulo de fuerzas de tensión que son transferidas al alveolo a través del ligamento periodontal. La tecnología a la fecha no ha sido capaz de duplicar exactamente esta interfase; los implantes osteointegrados tienen un efecto similar protector sobre el hueso subyacente nativo o reconstruido, esta es la razón por la cual la colocación de implantes dentales debe ser el componente de todos los planes de reconstrucción ósea alveolar en aras de la `preservación tridimensional del hueso alveolar.

\section{TRANSFERENCIA DE TEJIDO LIBRE MICROVASCULARIZADO}

La introducción de la transferencia de tejido microvascularizado al arsenal de técnicas disponibles ha facilitado la reconstrucción de la región maxilofacial y permite una mejor calidad de vida para los pacientes, permitiendo que estos tengan una mayor supervivencia así como posibilidades más amplias de reconstrucción. ${ }^{4}$

En 1975 Taylor y colegas describieron el injerto libre vascularizado de fíbula mientras Hidalgo en 1989 aplico dicha técnica para reconstrucción mandibular. ${ }^{5,6}$ y en 1978 Taylor describió la transferencia de cresta ilíaca como un colgajo osteomiocutáneo basado en irrigación sanguínea proveniente de la arteria y venas circunflejas, ${ }^{7,8}$ estos fueron avances muy importantes y sentaron las bases de la actual microcirugía. 
Los injertos libres se hicieron muy populares en la región de cabeza y cuello por la habilidad de transferir hueso y tejido blando en una fase al mismo tiempo de la resección con cifras de éxito predeciblemente altas. Es obvio que estos injertos incrementaron las opciones de disponibilidad de tejido así como la flexibilidad textura y color, todo esto en busca de alcanzar una reconstrucción y rehabilitación funcional para el paciente postoperado de resección tumoral, siendo sus mayores desventajas principalmente falta de altura, forma y grosor adecuados para recrear la intrincada anatomía mandíbulo-maxilar.

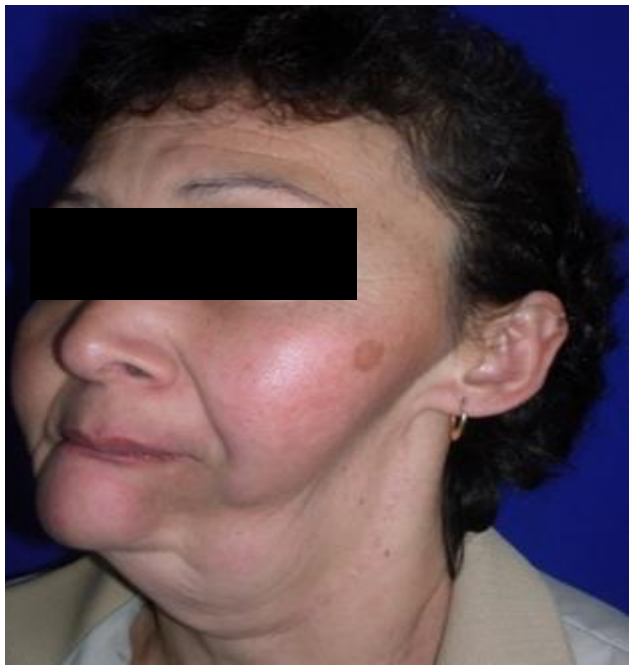

Fig.1 Vista frontal

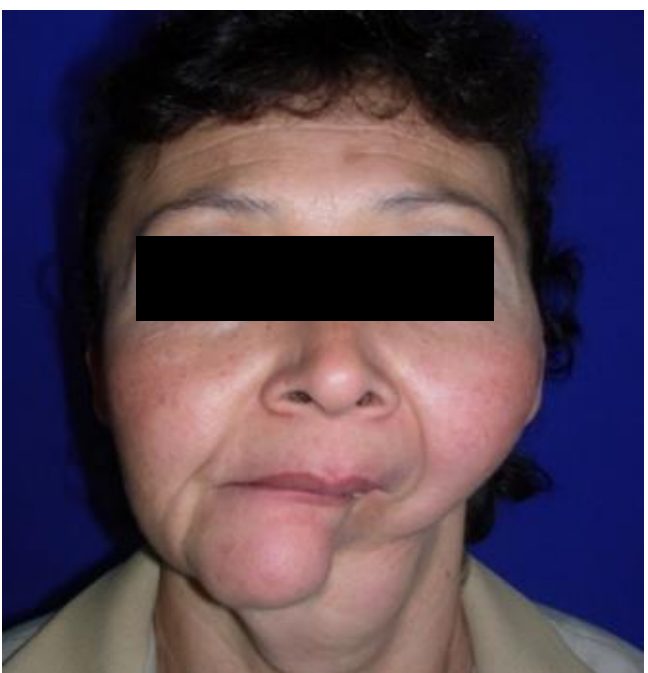

Fig. 2 Vista lateral tres cuartos del defecto

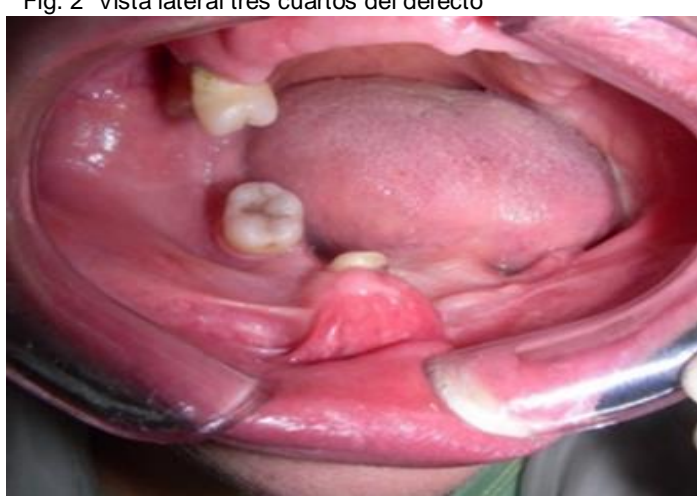

Fig. 3 Vista intraoral

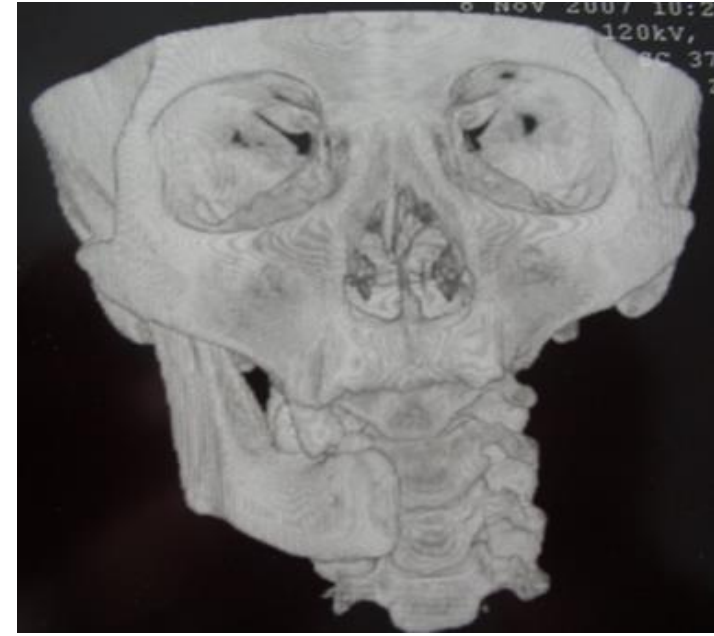

Fig. 4. Tomografía en 3D pre quirúrgica

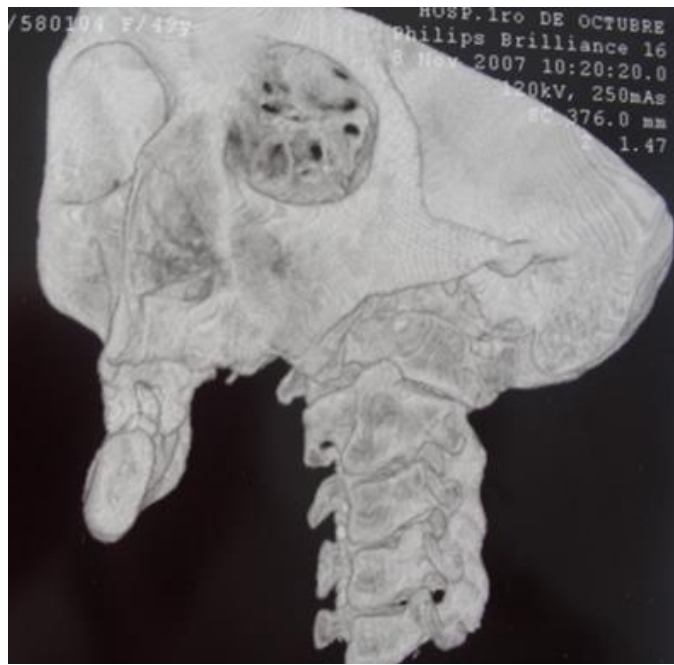

Fig. 5. Tomografía en 3D prequirúrgica.

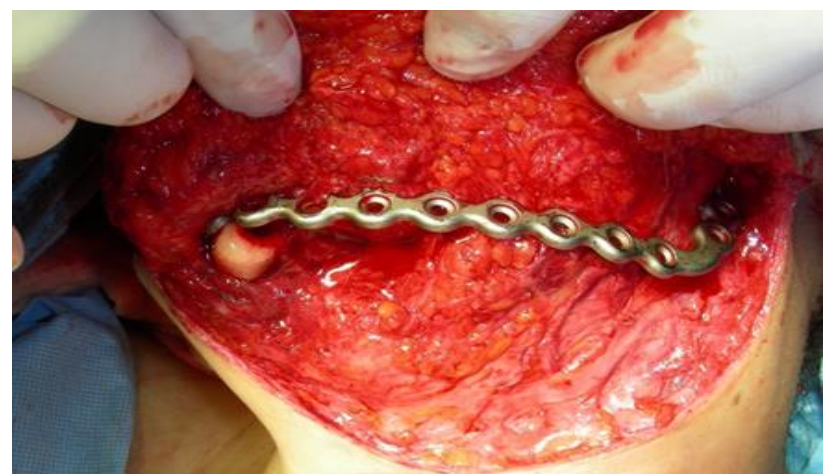

Fig. 6. Fotografía transquirúrgica colocación de placa de reconstrucción mandibular 


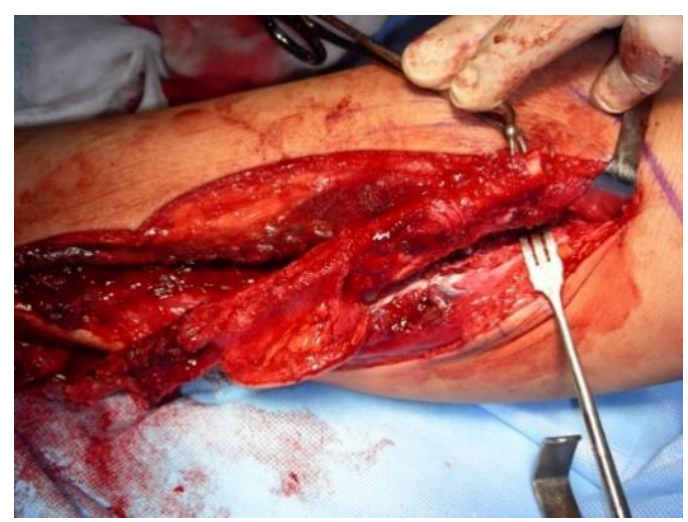

Fig. 7. Fotografía transquirúrgica zona donante

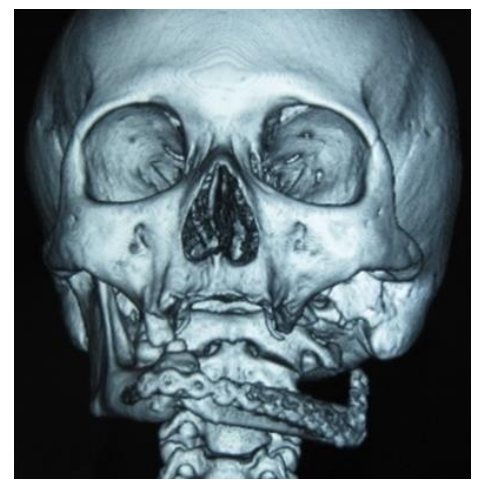

Fig. 8. Tomografía en 3D postoperatoria

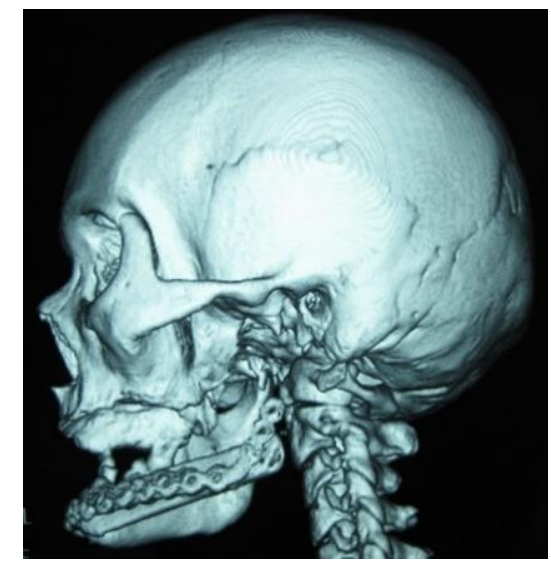

Fig. 9. Tomografía en 3D postoperatoria

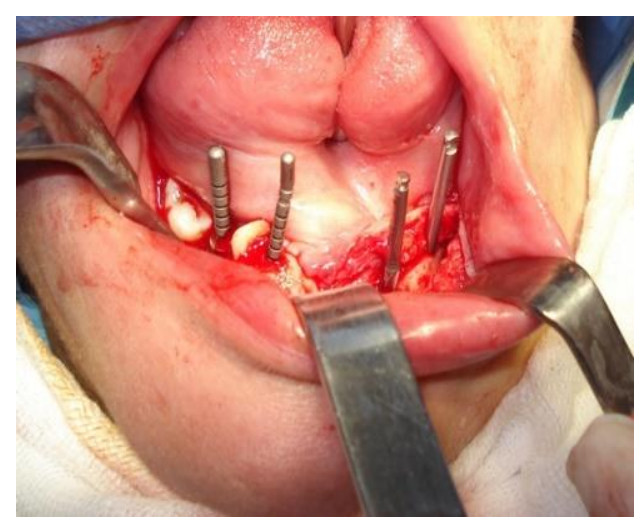

Fig. 10. Fotografía transquirúrgica, colocación de implantes dentales.

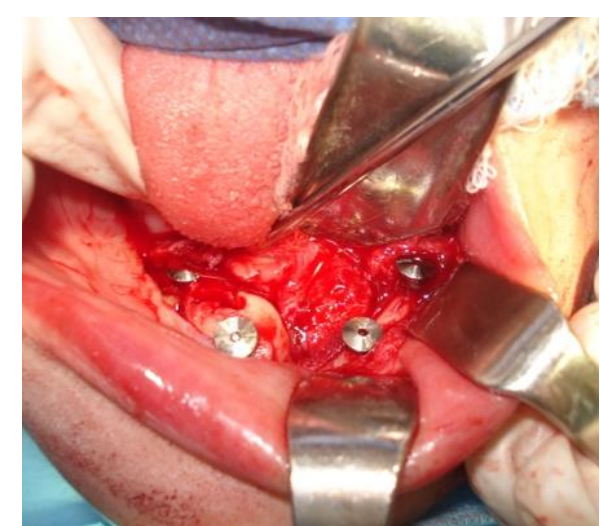

Fig. 11. Colocación de tapas de implantes dentales

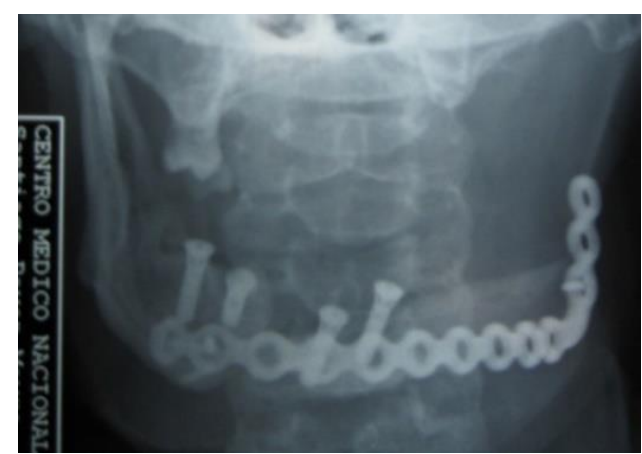

Fig. 12 Radiografía pósteroanterior de control.

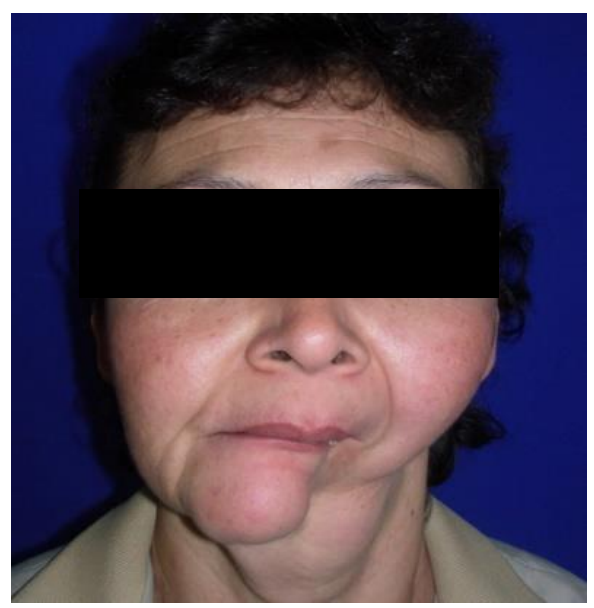

Fig. 13. Antes del tratamiento

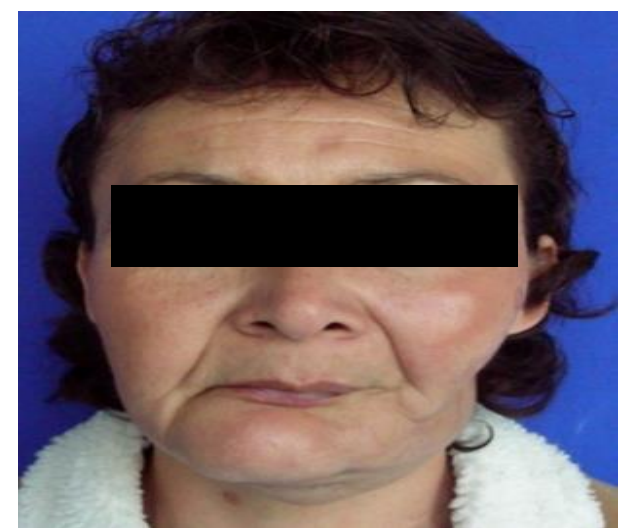

Fig. 14 Después (Control postoperatorio a los 6 meses 


\section{REFERENCIAS BIBLIOGRÁFICAS.}

1. Bloomquist $\mathrm{D}$, Turvey $\mathrm{T}$. Bone grafting and alternative procedures in Modern practice in orthognatic and reconstructive surgery 1992 Cap 28.

2. Enlow $\mathrm{DH}$, Kuroda $\mathrm{T}$, Lewis $\mathrm{AB}$. The morphological and morphogenetic basis for craniofacial form and pattern. Angle Orthod 1971; 41:161-88.

3. Moss ML. The primary role of functional matrices in facial growth. Am J Orthod 1969; 55:566-77.

4. Sullivan WG, Szwajkun PR. Revascularization of cranial versus iliac crest bone grafts in the rat. Plast Reconstr Surg 1991; 87:1105-8.

5. Taylor GI, Miller GDH, Ham FJ. The free vascularized bone graft: a free vascularized bone graft. Plast Reconstr Surg 1975; 55:533-44.

6. Hidalgo DA. Free fibula flap: a new method of mandibular reconstruction. Plast Reconstr Surg 1989; 84:719.

7. Taylor GI, Watson N. One-stage repair of compound leg defects with free, revascularized flaps of groin skin and iliac bone. Plast Reconstr Surg 1978; 61:494-506.

8. Taylor Gl. Reconstruction of the mandible with free composite iliac bone grafts. Ann Plast Surg 1982; 9:361-76. 